MATEC Web of Conferences 44, 02084 (2016)

DOI: $10.1051 /$ matecconf/20164402084

(C) Owned by the authors, published by EDP Sciences, 2016

\title{
Regional differences of urban construction land in Jiangsu province, China
}

\author{
Xiu Lu ${ }^{1}$, Jia Li ${ }^{1, a}$, Fei Tao ${ }^{2}$ and Ping Duan ${ }^{1}$ \\ ${ }^{1}$ School of Tourism and Geographical Sciences of Yunnan Normal University Yunnan, China \\ ${ }^{2}$ School of Geographical Sciences of Nantong University Jiangsu, China
}

\begin{abstract}
Jiangsu province is a typical area of the urban extension problems. Urban construction land has been the major area of city research. And more and more people pay attention to this matter. In this paper, regional differences of urban construction land in Jiangsu province, China were analyzed. Firstly, this work is based on remote sensing images as the main data source in 2010. The spectral differences of soil cover type of waters, forest land, construction land, unused land, cultivated land were explored. Secondly, three kinds of index methods (soil adjusted vegetation index, modified normalized difference water index and the ratio resident-area index) were used to extract the information of urban construction land. Finally, urban construction land was segmented by administrative boundary information of Jiangsu Province, China. And, different areas of the urban construction land, non-urban construction land and cultivated land were compared and analyzed. The experimental results from the urban construction scale show that, in 2010, the south of Jiangsu is greater than the north of Jiangsu, and the north of Jiangsu is greater than the central of Jiangsu. From the point of proportion of cultivated land: the central of Jiangsu is greater than the north of Jiangsu, and the north of Jiangsu is greater than the south of Jiangsu.
\end{abstract}

\section{Introduction}

The increasing urban construction land is the significant identification of the changes of landscape structure of the rapid development. The dynamic process not only significantly changed the 1 overall landscape layout of city zone. At the same time it also has a significant impact on the edge of the city's agricultural distribution and the layout of natural humanities' landscape. Our country is in the stage of rapid urbanization. And Jiangsu province is the typical area of urban expansion in our country [1]. Urban land has been the main direction of urban studies. With the development of remote sensing and GIS technology, the scholars in domestic and abroad have done a lot of research on some cities in Jiangsu province and the improvement of the research methods in recent years. Ran Jiang etc (2007) adopted the method of supervised classification and visual interpretation to analyse Lianyungang city's extension and encroach on the surrounding land from 1978 to 2005 [2]. Wang lei (2008) based on the data of urban construction land area in the past years of Jiangsu province, analysing the change of time and space of the construction land expansion and exploring its effect mechanism. Zhang Xuefeng etc (2011) used TM3 and TM2 two bands transplanting in normalized construction index (NDBI) method and he combined it with NDBI to extract the urban construction land. He set a define method to be suitable for the remote sensing classification establishment and which can acquire three cities built up area of Suzhou, Wuxi and Changzhou [3]. Wu XIU (2012) used RS and GIS spatial information extraction, spatial analysis technology and theory of cellular automata to analyse and study the strength, scale and direction of urban spatial extension characteristics, dynamic mechanism. Xu Hanqiu (2005) chosen Fuzhou city thematic enhanced images as the research data, and he selected the Normalized difference construction index, soil vegetation index adjustment and correction normalized difference water index and so on to analyse the spectral characteristics. And then extract the information of urban construction land area, the main method is the rules of logic criterion [4]. Wu Hongan(2006) proposed a new method of extracting information, and he used the ratio resident index method (RRI). This method is collecting the sampling points of images of the study area and using the $\mathrm{DN}$ value of the image to be able to quickly extract urban building land information [5].

This paper is using remote sensing images and it is based on remote sensing and GIS to analyse the land used for urban construction in 2010 of the whole Jiangsu province. This paper is also combined with Jiangsu province administrative boundary data to obtain the

${ }^{a}$ Corresponding author: Li Jia $(\bowtie)$

School of Tourism and Geographical Science, Yunnan Normal University, Kunming, China

e-mail: keguigiser@163.com 
partition of land used for urban construction and it is accessed to different parts of the area, distribution features of urban construction land.

\section{Research area and data}

\subsection{Research area}

Jiangsu province is simply called "Su". Its location is in the eastern coastal center of mainland China. Its latitude and longitude is: $116^{\circ} 18^{\prime} \sim 121^{\circ} 57^{\prime} 116^{\circ}$ east longitude, $30^{\circ} 45^{\prime} \sim 35^{\circ} 20^{\prime}$ north latitude. In Jiangsu province, its area is 102600 square kilometers and its area occupies $1.06 \%$ of the territory of China. Its per capita land area is the least of all the provinces in China. Jiangsu is near the ocean and near the sea, adjoining the yellow sea in the east and the Pacific Ocean. It is geographically across north and south and its climate and vegetation also has the characteristics of the south and the north. Its border is adjacent to Shandong, Zhejiang, close to the Anhui province and Shanghai City. To 2015, Jiangsu province has administered a deputy provincial city (Nanjing), 12 district cities (Wuxi, Xuzhou, Changzhou, Suzhou, Nantong, Lianyungang, Huaian, Yancheng, Yangzhou, Zhenjiang, Yangzhou, Taizhou and Suqian).

\subsection{Data source}

\subsubsection{Remote sensing image data}

Because LANDSAT7 ETM + airborne scanning line corrector occurred major fault after May 2003. This paper selects the LANDSAT5 TM data as data sources. The 23 remote sensing images are selected for Mosaic. Due to the much image data of Jiangsu province, cloud cover, vegetation growth, some data loss and so on, some 2010 cannot use the image data, 2009 image data instead with the same region. Finally 23 images are used for mosaic which respectively including 10 images of 2009 and 13 images of 2010.

\subsubsection{Administrative division data}

Political map of Jiangsu province and reference in 2009 and 2010 yearbook data of Jiangsu province and other relevant information.

\subsubsection{Data pre-processing}

The 23 images are downloaded from Geospatial data cloud and to conduct band combination, image correction, image matching, image of administrative boundary cutting and so on in the data preprocessing.

\section{Research method}

\subsection{Analysis of spectral information data}

Because different land types have different spectral brightness values, this paper is based on predecessor-Che Feng [6] to analyse the characteristic of the spectral difference which can extract the construction land information. Choose a number of sampling points of forest land, water land, unused land, urban land and the cultivated land and so on, five land-cover types. Draw the curve between the average Digital Number and the land type class band. The general image is shown in Fig.1.

Fig. 1 showing the construction land and unused land has the same change trend. Cultivated land and forest land have a similar spectral curve trend. Water has its unique spectral curve. According to the spectrum analysis of DN values, therefore, it can be simply concluded 5 classes into 3 classes: vegetation, water, and land for construction.

Secondly, it has clearly seen that in Fig.1, various classes of each band spectrum intensity is not completely consistent to another one. So we can find the gap between the other classes and the construction land and we can clearly distinguish the construction land from the information of the other classes. Now order different wave bands of different classes and the result is as follows:

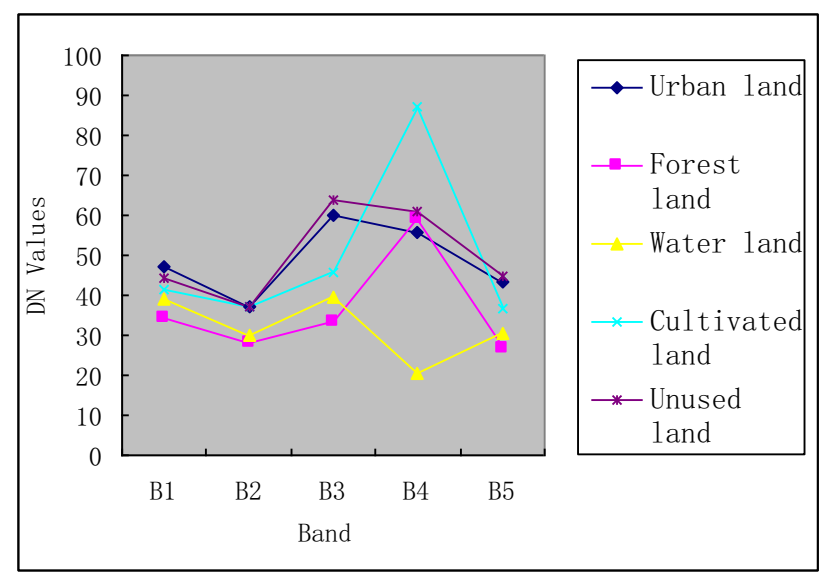

Figure1. Land type of spectrum curve

Cultivated land: band $6>$ band $1>$ band $4>$ band $5>$ band $3>$ band $2>$ band 7

Forest land: band $6>$ band $1>$ band $4>$ band $5>$ band $3>$ band $2>$ band 7

Water land: band $6>$ band $1>$ band $3>$ band $2>$ band $4>$ band $5>$ band 7

Unused land: band $6>$ band $5>$ band $1>$ band $7>$ band $3>$ band $4>$ band 2

Construction land: band $6>$ band $1>$ band $5>$ band $3>$ band $4>$ band $2>$ band 7

After sorting, it is found that the cultivated land and the forest land satisfying: band $4>$ band3. Unused land and construction have a relatively similar spectrum curve of the change trend. The values of their band5 are greater than band4. According to the spectral characteristics, we adopt previous information extraction and classification model. It is a method of SAVI, MNDWI and RRI. The three-index exactly play a role of extracting the most critical urban land use types: vegetation, construction and water. Introduction of Soil Adjusting Vegetation Index can be used to distinguish between vegetation and urban construction land. And then introduction of Modified 
Normalized Difference Water Index can distinguish between water and urban construction land. But because the spectral characteristics of the unused land and the urban construction land are very similar, the information of extracting contains the construction land information. Therefore it has to remove unused information in the end. From that we can obtain the complete and accurate information of the construction land.

\subsection{Extraction of vegetation information}

To reduce the interference of background object, on the basis of NDVI, Huete proposed a SAVI index namely soil adjusting vegetation index [7]. Its formula is:

$\mathrm{SAVI}=[(\mathrm{TM} 4-\mathrm{TM} 3)(1+\mathrm{L})] /(\mathrm{TM} 4+\mathrm{TM} 3+\mathrm{L})$

In the formula, $\mathrm{L}$ is a factor to adjust the soil. 0 is used for high vegetation coverage and the high richness. $\mathrm{L}$ is used for low vegetation coverage and the low richness. In general, when $\mathrm{L}$ takes 0.5 , it can better decrease the difference of soil.

\subsection{Extraction of water information}

$\mathrm{Xu}$ Hanqiu in 2005 put forward the Modified Normalized Difference Water Index. Operating the ratio between the band 2 and the band 5 and then to extract water body information. Its formula is [8]:

$$
\text { MNDWI }=(\text { TM2-TM5 }) /(\text { TM+TM5 })
$$

It can be concluded, Modified Normalized Difference Water Index (MNDWI) extraction effect is superior to the Normalized Difference Water Index (NDWI). Using MNDWI extracts the scope of the water whose effect to the actual situation is superior to NDWI.

\subsection{Extraction of the construction land information}

Yang Shan in 2000 put forward a Normalized Difference Vegetation Index [9], it is a precursor to NDBI index. Later Zha Yong and others later changed it to the Normalized Difference Construction Index. Its formula is:

$$
\text { NDBI }=(\text { TM5-TM4) / (TM5+TM4) }
$$

Band5 and band 4 are possibly all zero in this formula, so we need to add 0.001 in the denominator. Analysing again, construction land near-infrared wave band is less than the infrared wave band. The same applies to unused land and forest land. So the extraction of the construction land information also contains a certain amount of unused land information and forest land information.

\subsection{Division of unused land and urban construction land information}

The above extraction of construction land inside contains a lot of unused land information. So we must remove unused land information. We can use RRI $=\mathrm{B} 1 / \mathrm{B} 4$ to conduct the band calculation [5].

\section{The extraction results and the analysis of regional differences}

\subsection{The extraction results}

Use the SAVI to extract the vegetation information. Use the MNDWI to extract the water information. Use the RRI to apart the construction land from the unused land. It can generate three images including SAVI, MNDWI and RRI in the above process. Now we respectively merge the images as three new bands. It can be used to separate water, urban construction land and unused land and it can reduce data redundancy. The result is shown in Fig.2.Using the maximum likelihood method to pick up the urban land, like Fig.3.Through calculation, the area of urban land and non-urban land use situation in the following Table1.

Overall, the urban construction land in Jiangsu province occupies $1 / 5$ of the total area of Jiangsu province. The remaining four categories: forest land, unused land, water and cultivated land accounts for $4 / 5$. And then divide the Jiangsu province into 13 cities. Then analyse each prefecture level city construction land and preliminarily analyse the rationality of urban planning.

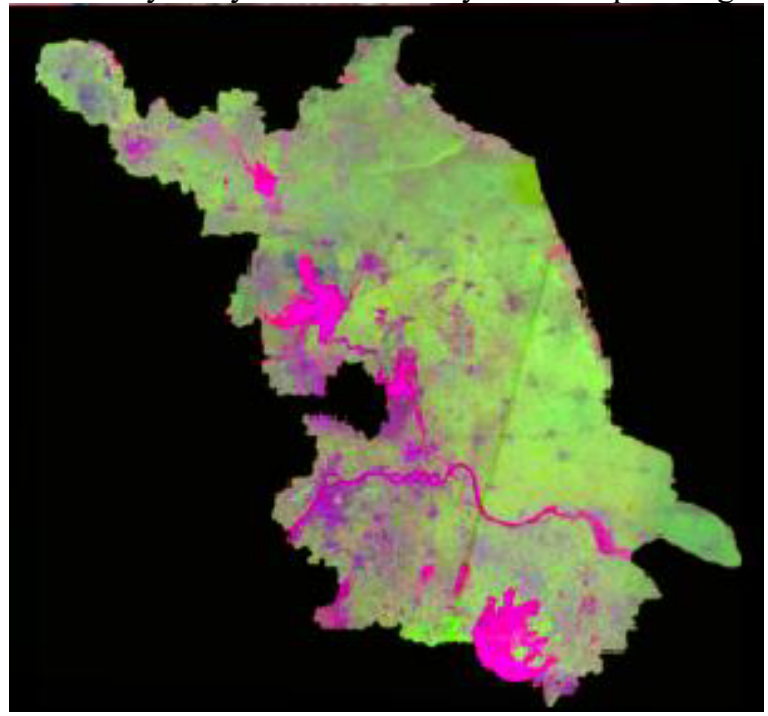

Fig 2. The three index composition image

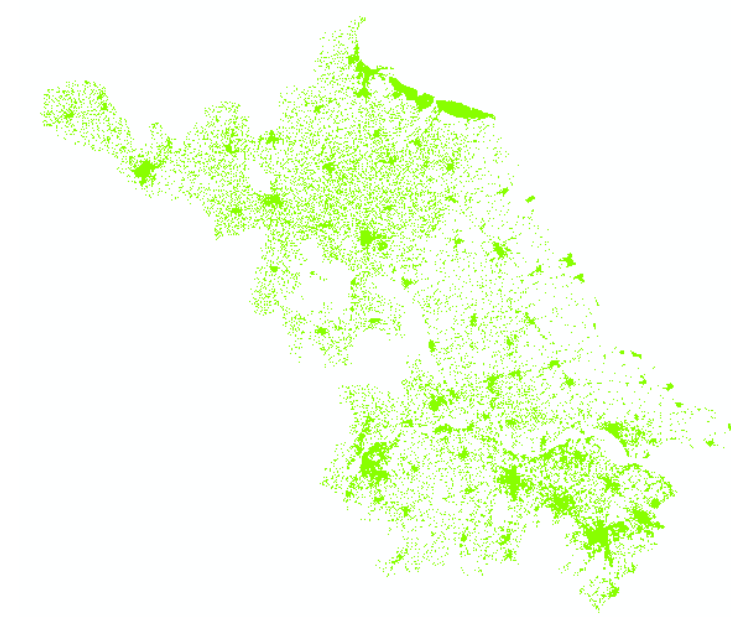

Fig 3. Urban land extraction results in Jiangsu province 
Table1. Land use type area in Jiangsu province

\begin{tabular}{cc}
\hline Land use types & Area/km² \\
\hline Urban construction land & 20060.699 \\
$\begin{array}{c}\text { Non-urban construction land } \\
\text { Regional land use in Jiangsu } \\
\text { province }\end{array}$ & 84038.707 \\
\hline
\end{tabular}

\subsection{Regional difference analysis}

Crop the land use type picture in Jiangsu province. And divide the figure into 13 cities, namely: Zhenjiang, Changzhou, Wuxi, Nanjing, Suzhou (southern area of Jiangsu), Nantong, Taizhou, Yangzhou (middle area of Jiangsu), Xuzhou, Lianyungang, Suqian, Yancheng, Huaian (northern area of Jiangsu). Use three-index method to extract 13 cities information such as the urban construction land. In software arcgis10.0, we can compute area. It can draw various land types area of the cities (including urban construction land area, cultivated land area and so on). The result shows in Table 2.

Table 2. Land type area of 13 cities in Jiangsu province

\begin{tabular}{cccc}
\hline $\begin{array}{c}\text { Prefecture- } \\
\text { level city }\end{array}$ & $\begin{array}{c}\text { Urban } \\
\text { construction } \\
\text { land area } \\
\text { (Unit:km²) }\end{array}$ & $\begin{array}{c}\text { Cultivated } \\
\text { land area } \\
\left.\text { (Unit:km }^{2}\right)\end{array}$ & $\begin{array}{c}\text { Total area } \\
\text { (Unit: } \mathbf{k m 2})\end{array}$ \\
\hline Xuzhou & 2514.8899 & 7632.8198 & 11102.03 \\
Lianyungang & 1864.0601 & 4670.0801 & 7309.387 \\
Suqian & 2005.4301 & 5058.0898 & 8567.689 \\
Huaian & 1951.53 & 5714.6099 & 9972.295 \\
Yancheng & 1994.9301 & 11664.9 & 15334.89 \\
Yangzhou & 1051.14 & 4211.0298 & 6609.79 \\
Taizhou & 995.82898 & 4197.9302 & 5783.223 \\
Nantong & 976.66803 & 7373.96 & 8901.446 \\
Zhenjiang & 728.70001 & 2358.0901 & 3826.502 \\
Changzhou & 926.98999 & 2506.02 & 4384.301 \\
Wuxi & 1215.36 & 1946 & 4602.012 \\
Suzhou & 2206.54 & 2949.6101 & 8405.863 \\
Nanjing & 1551.78 & 3490.46 & 6535.961 \\
\hline
\end{tabular}

To sum up, Xuzhou, Huaian, Yancheng, Suzhou and Suqian cities' land area are the largest all over the 13 cities' urban construction land area in Jiangsu province. Five cities have four cities are northern regions. According to related statistics, all over the Jiangsu province, the north of Jiangsu economy is relatively less than middle and southern area of Jiangsu. Urban land area is large, does not represent the whole area proportion is large too (As shown in Fig. 4). The data shows in figure 5, Suzhou and Wuxi urban land area of total area proportion are the most. Southern area of urban land proportion ranges between $19 \% \sim 26 \%$ and there is not big difference among them. Yancheng's urban land area is large, but the urban land area only $t$ account for $13 \%$ of all over the whole area.

Overall, the construction land is the largest proportion of southern region of Jiangsu. Secondly, northern region, middle region is the lowest. In contrast to the urban construction land proportion, larger proportion is the middle region, northern regions secondly, followed by a relatively low proportion is southern region in the 13 cities. (As is shown in Fig.5).

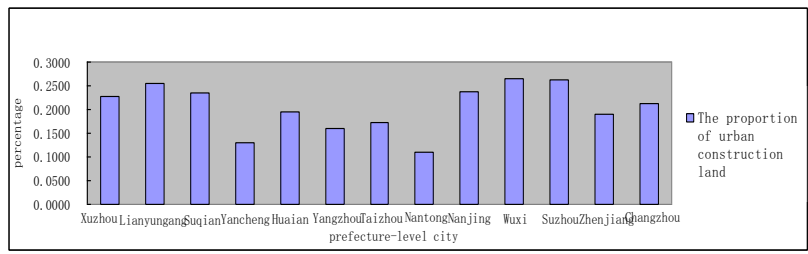

Fig 4. Proportion of urban construction land use of prefecturelevel cities in Jiangsu province

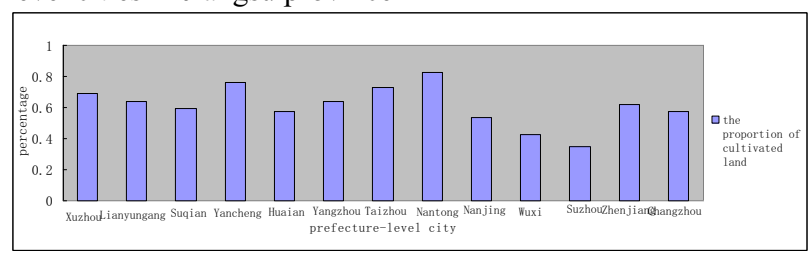

Fig 5. Proportion of cultivated land use of prefecture-level cities in Jiangsu province

Therefore, throughout the proportion of the level city in Jiangsu province and under the other land classes share a similar situation, proportion of cultivated land area is opposite to urban construction land. So, from this point, it laterally reflects the city's expansion and development based on the loss of arable land and transformation. The geographic space main present a characteristic of middle area is greater than the southern Jiangsu and greater than the northern Jiangsu area from the overall coordination between the level of economic development in Jiangsu province and the city level of intensive land use type.

\section{Conclusions}

This paper is based on the multiphase LANDSAT TM data as the data source. Select appropriate extraction method to extract the information of urban construction land through exploring water areas, forest land, construction land, unused land and cultivated land and so on five spectral differences of soil cover types. Make use of Jiangsu province administrative boundary information to partition for the urban construction land. Compare different parts of the city construction land area, difference of distribution characteristics. It can reveal the area of urban construction land in Jiangsu province, morphological differentiation and the pattern of the spatial distribution characteristics. The specific conclusions are as follows:

1. This paper uses SAVI, MNDWI, RRI three indices for urban construction land information extraction. The result shows that the method to extract the area size is similar to the known area of the city planning of the cities. It can be illustrated that the three index extraction method is a high extraction precision and it is a kind of relatively accurate urban land information extraction technology.

2. Jiangsu province has a total area of 104099.406 square kilometers, of which the urban construction land area of 20060.699 square kilometers, accounting for $1 / 5$ of the total area. Divide Jiangsu province into three regions: northern area, middle area and southern Jiangsu. Southern area five regional average proportion of urban land is: 0.208281 . Southern Jiangsu five regional average proportion of urban land is: 0.233177 . Middle region of the five regional average proportion of urban land is: 0.14698 . The south of Jiangsu is greater than northern 
region and is greater than the middle region. But from the perspective of cultivated land, north Jiangsu five regional cultivated land proportion is 0.6501 on average. Five regional average proportion of cultivated land in the south of Jiangsu is 0.49913 . Middle five regional average proportion of cultivated land is 0.73046 . The middle region is greater than the northern region and is greater than the southern region.

All in all, the government should grasp the coordination of urban development and land use types. And we need to give full play to regional advantages and to improve land use efficiency. At the same time, giving play to the role of the government's macroeconomic regulation and control and to promote coordinated development of regional economy orderly is an extremely urgency.

\section{References}

1. Q.M. Wu and Lv H, "Jiangsu province for nearly 30 years of land use change analysis of the regional differences," The Earth Information Science Journal, 05:5670-5676, 2009.

2. Q. Ran and C.Q. Ke, "Lianyungang urban expansion and its driving force analysis," Anhui Agricultural Science, 03:725-727, 2007.

3. X.F. Zhang, Z.W. He, R. Wang and D.H. Zhang, "Su, Xi, Chang built up area used remote sensing method to extract and the analysis of urban expansion," Science of Surveying and Mapping, 02:113-115, 2011.

4. H.Q. Xu, "It is based on the spectral characteristics and normalized index to the urban building land information extraction," Geographical Research, 24(2):311-320, 2005.

5. H.A. Wu, J.J. Jiang and H.L. Zhang, "Residents ratio index apply in the information extraction in cities and towns," Journal of Nanjing Normal University (Natural Science Edition), 118-121, 2006.

6. F. Che and H. Lin, "Urban construction land remote sensing information extraction method research," Science of Surveying and Mapping, 04:97-99, 2010.

7. A.R. Huete, "A soil adjusted vegetation index (SAVI)," [J]Remote Sensing of Environment, 25(3): 295-309, 1988.

8. H.Q. Xu, "Using the modified normalized difference water index (MNDWI) to extract water body information research," Journal of Remoting Sensing, 9(5):589-585, 2005.

9. S. Yang, "Developed areas of urban and rural human settlements of information extraction and fractal research - in Wuxi city as an example," Journal of Geographical, 55(6):671-678, 2000. 